\title{
A Theoretical Foundation and a Method for Document Table Structure Extraction and Decompositon
}

\author{
Howard Wasserman, Keitaro Yukawa, Bon Sy, Kui-Lam Kwok, and \\ Ihsin Tsaiyun Phillips \\ Department of Computer Science \\ Queens College, the City University of New York \\ 65-30 Kissena Boulevard \\ Flushing, New York 11367-1597 \\ howard@umedia.cs.qc.edu, yukawa@picasso.cs.qc.edu, bon@bunny.cs.qc.edu, \\ kwok@ir.cs.qc.edu, ihsin_phillips@qc.edu
}

\section{Introduction}

The algorithm described in this paper is designed to detect potential table regions in the document, to decide whether a potential table region is, in fact, a table, and, when it is, to analyze the table structure. The decision and analysis phases of the algorithm and the resulting system are based primarily on a precise definition of table, and it is such a definition that is discussed in this paper. An adequate definition need not be complete in the sense of encompassing all possible structures that might be deemed to be tables, but it should encompass most such structures, it should include essential features of tables, and it should exclude features never or very rarely possessed by tables.

The remainder of the paper is organized as follows. The table definition is obmitted due to the page limitation. Please contact the author for detail. We describe the propose table region detection algorithm in details Section 2, and we conclude with our future directions in Section 3.

\section{The Proposed Table Region Detection Algorithm}

Typical document image analysis and recognition systems consist of the following main modules - scanning/digitization, page physical layout analysis, page logical layout analysis, and the output formatting modules. Within the page layout analysis module are typically included sub-modules that extract text-words, text-lines, text-paragraphs, text-columns, as well as sub-modules for non-text region extraction and labeling. In this section we outline our proposed algorithm for table region detection which will become a component of an overall document table-understanding system within any OCR system. Our algorithm takes as input a set of word boxes, the result of the word segmentation technique in [11], produces from this set an initial set of "black boxes" which is subsequently

D. Lopresti, J. Hu, and R. Kashi (Eds.): DAS 2002, LNCS 2423, pp. 291294 2002

(C) Springer-Verlag Berlin Heidelberg 2002 
processed by the following modules discussed in this section: word-box consolidation and text-line elimination module, vertical-range module, horizontal-range module, and table-region extraction module, yielding a final set of potential table-region bounding boxes which constitutes suitable input for a table decomposition algorithm.

\subsection{Problem Statement}

Given is a set of word boxes $\mathbf{W}=\left\{b_{i}: b_{i}=\left(x_{i}, y_{i}, w_{i}, h_{i}\right)\right\}$ where $\left(x_{i}, y_{i}\right)$ is the coordinates of the upper-left corner of the word box $b_{i}, w_{i}$ is the width of $b_{i}$, and $h_{i}$ is the height of $b_{i}$, with the requirement that $0 \leq x_{i}, w_{i} \leq$ width of a given document page $P$ and $0 \leq y_{i}, h_{i} \leq$ height of the given document page $P$. The problem is to compute the collection of potential table regions on the given document page: $\mathbf{T}=\left\{T_{i}: T_{i} \subseteq \mathbf{W}, T_{i}\right.$ a potential table region $\}$ such that for each pair $i, j$ with $i \neq j, T_{i} \cap T_{j}=\emptyset$.

\section{$2.2 \quad$ Test Data}

The initial test data set for the algorithm will be taken from recent proceedings of scientific conferences that are available to the public in PDF file format. These PDF files will be converted into TIFF image format. We intend to collect at least 100 instances of such test data, some of which will include tables, and some not, to insure objective testing.

\subsection{Overall Description}

The input to the algorithm is a set $\mathbf{W}$ of word boxes. The output of the algorithm is the set of potential table regions. The algorithm consists of the following modules in the stated order: a word-box consolidation and text-line elimination module, a vertical-range module, a horizontal-range module, and a table-region extraction module.

The set, $\mathbf{W}$, of word boxes is passed as input to a word-box consolidation and text-line elimination module, producing a reduced set of (combined) word boxes called "black boxes". This set of black boxes is then passed to a vertical-range module which, using horizontal projection profiles, produces vertical ranges of potential table regions. For each vertical range produced, the set of black boxes in it is passed to a horizontal-range module which, using vertical projection profiles, produces horizontal ranges of potential table regions. The computed vertical and horizontal ranges are passed to a table-region extraction module, which produces the final set of potential table-region bounding boxes.

\subsection{Word-Box Consolidation and Text-Line Elimination}

Let $\mathbf{W}$ be the input set of word boxes. For each $b_{i} \in \mathbf{W}$ that has a right neighbor, compute its right gap box $g_{i}$. Let $\mathbf{R G}=\left\{g_{i}\right\}$ be the set of all right gap boxes. 
Small, inter-word gap boxes are then eliminated as follows. Compute the gap-width histogram to determine a threshold value $\rho$ for inter-word gap widths. Consider each word box to be an initial "black box". Then, iteratively, delete each $g_{i} \in \mathbf{R G}$ whose width is less than the threshold $\rho$, and for each deleted gap box $g_{i}$, horizontally merge the two adjacent black boxes together with the gap box $g_{i}$ into a longer black box. Let the resulting set of black boxes be $\mathbf{B}$.

Black boxes for regular text lines are subsequently eliminated as follows. Compute a histogram of the widths of black boxes in $\mathbf{B}$. Then determine a threshold from the histogram to eliminate black boxes for regular text lines - in so doing, the following observation may be used: table entries are normally less than half the average width of text lines. Then eliminate the black boxes in $\mathbf{B}$ whose width is greater than the threshold. (Remark: If a black box has a width not greater than the threshold but follows a run of one or more text-line black boxes, it is normally not a table entry.)

\subsection{Computation of Vertical and Horizontal Ranges of Potential Table Regions}

The fundamental idea behind the following two modules is to compute vertical and horizontal projection profiles of the black boxes, and then detect patterns of profiles suggesting potential table regions (PTRs) using finite automata.

Vertical Range Modules. Compute a horizontal projection profile, HP, of the black boxes in $\mathbf{B}$.

Then do the following preprocessing to clean up the horizontal profile by eliminating short, noisy black boxes: Compute a histogram of lengths of runs of 1's (here and subsequently "1" symbolically denotes any positive number). Compute a threshold, $\rho$, such that those runs of 1's of length less than $\rho$ should be eliminated. Eliminate those short runs of 1's by turning the 1's to 0's. Let the resulting, cleaned up, horizontal profile be $P^{\prime}$.

In the next step, parameters for a finite automaton to detect vertical ranges of potential table regions (PTRs) are computed: Compute a histogram of runs of 1's from $P^{\prime}$, and determine the parameter values pertaining to heights of black boxes, to be used in the finite automaton to detect PTRs. Compute a histogram of runs of 0 's from $P^{\prime}$, and determine the parameter values pertaining to heights of inter-line, inter-row, and inter-table white gaps, to be used in the finite automaton to detect PTRs.

Construct the finite automaton using the computed parameters, and then apply the finite automaton to detect the vertical ranges of PTRs.

Horizontal Range Modules. For each vertical range of a PTR detected, do the following:

Compute a vertical projection profile, VP, for the black boxes in B. Do a clean-up processing on VP similar to that done in the vertical-range detection module. Compute histograms of lengths of runs of 0's and 1's, and obtain 
thresholds. Apply a "non-maximum suppression" technique using the thresholds to obtain an accentuated vertical profile. Apply a finite automaton technique to detect columns and column boundaries of the PTR.

\subsection{Table Region Extraction}

Using the vertical and horizontal PTR ranges computed, return the set of word boxes falling within the intersection of the two ranges.

\section{References}

1. Y. Wang, I. T. Phillips, and R. Haralick. Automatic table ground truth generation and a background-analysis-based table structure extraction method. Sixth International Conference on Document Analysis and Recognition(ICDAR01), pages 528-532, September 2001.

2. E. Green and M. Krishnamoorthy. Model-based analysis of printed tables. Proceedings of the 3rd ICDAR, pages 214-217, August 1995.

3. J. H. Shamilian, H. S. Baird, and T. L. Wood. A retargetable table reader. Proceedings of the 4th ICDAR, pages 158-163, August 1997.

4. T. G. Kieninger. Table structure recognition based on robust block segmentation. Document Recognition V., pages 22-32, January 1998.

5. T. Kieninger and A. Dengel. Applying the t-rec table recognition system to the business letter domain. Sixth International Conference on Document Analysis and Recognition(ICDAR01), pages 518-522, September 2001.

6. B. Klein, S. Gokkus, T. Kieninger, and A. Dengel. Three approaches to "industrial" table spotting. Sixth International Conference on Document Analysis and Recognition(ICDAR01), pages 513-517, September 2001.

7. K. Zuyev. "Table image segmentation". Proceedings of ICDAR, Germany, 1997. pp.705-708, 1997.

8. J. Hu, R. Kashi, D. Lopresti and G. Wilfong. A system for understanding and reformulating tables. Proc. 4th IAPR Intl. Workshop on Document Analysis Systems - DAS'2000. pp.361-372, 2000.

9. J. Hu, R. Kashi, D. Lopresti, and G. Wilfong. Medium-independent table detection. SPIE Document Recognition and Retrieval VII, pages 291-302, January 2000.

10. J. Liang, I. T. Phillips, and R. M. Haralick. Consistent partition and labeling of text blocks. Journal of Pattern Analysis and Applications, 3:196-208, 2000.

11. Yalin Wang, Ihsin. T. Phillips, Robert M. Haralick, Statistical-based Approach to Word Segmentation. Proceedings of the 15th International Conference on Pattern Recognition(ICPR2000), Barcelona, Span, September 3-7, 2000, p.555-558.

12. Jisheng Liang, Ihsin. T. Phillips, and Robert M. Haralick, "An Optimization Methodology for Document Structure Extraction on Latin Character Documents", the Journal of IEEE Transactions for Pattern Analysis and Machine Intelligence, 2000 .

13. Jisheng Liang, Ihsin. T. Phillips, and Robert M. Haralick, "Performance Evaluation of Document Structure Extraction Algorithms", the Journal of computer Vision and Image Understanding, 2000. 